Wien klin Mag 2018 · 21:1

https://doi.org/10.1007/s00740-018-0212-7

Online publiziert: 15. Januar 2018

(c) Springer-Verlag GmbH Austria, ein Teil von Springer Nature 2018

CrossMark
Verena Kienast

SpringerMedizin, Wien, Österreich

\section{Akademisierung versus Bildung}

\section{Nicht alles ist quantifizier- und messbar}

Für zahlreiche Universitätsstudien gibt es sie schon, bei künstlerischer akademischer Ausbildung wurde sie nie wirklich in Frage gestellt und für den Besuch des Gymnasiums wird sie nun wieder vorgeschlagen: Die Aufnahmeprüfung als Eintrittspforte in spezielle Bereiche hat wieder Hochsaison. Es gibt freilich eine Menge Argumente dafür ebenso wie dagegen und auch eine Reihe von ,ja, aber" oder „wenn, dann“-Begründungen. Für einfache Antworten sind unsere über Jahrhunderte gewachsenen Bildungs- und Ausbildungssysteme mittlerweile schon $\mathrm{zu}$ komplex geworden und für wirklich radikale Änderungen fehlt ohnedies der Mut. In Deutschland ist man immerhin mittlerweile draufgekommen, dass die Leistungsschau über das absolvierte Abitur in Form eines Notendurchschnitts - vulgo numerus clausus - als dominante Eintrittskarte für das Medizinstudium auch nicht das Gelbe vom Ei ist. Es widerspricht, ebenso wie die Wartezeit, als Kriterium dem Grundrecht auf „gleicher Teilhabe am staatlichen Studienangebot" urteilte das Verfassungsgericht kurz vor Weihnachten.

\section{Soft Skills lassen sich schwer messen}

Das zugrundeliegende Problem für derartige Zugangsbestimmungen ist vor allem die begrenzte Zahl an Studienplätzen, die eine sinnvolle Betreuung der Studierenden möglich machen soll. Also muss eine Auswahl erfolgen. In unserer Leistungsgesellschaft will man natürlich die Besten. Und wie diese zu finden sind, muss sich irgendwie quantifizierbar und messbar machen lassen. Kein leichtes Unterfangen. Die sogenannten soft skills, auf die ja doch mittlerweile ein bisschen Licht fällt, lassen sich eben nicht so gut mes- sen, und nicht jede im Prüfungsgespräch gegebene Antwort muss ehrlich sein, sondern kann auch einfach den wahrgenommenen Erwartungen entsprechend gegeben werden.

Durchaus im Widerspruch $\mathrm{zu} \mathrm{Zu-}$ gangsbeschränkungen steht dabei das Credo, dass die Akademisierungsquote angehoben werden soll. Ein Ziel, das sich zwar messen lässt, aber für sich genommen noch ziemlich inhaltsleer ist. Die Absolvierung des Studiums - welches auch immer - und die Erlangung eines akademischen Titels sagt noch ziemlich wenig über die grundlegenden Kompetenzen, die Diskurs- und Reflexionsfähigkeit und die Fähigkeit, komplexe Inhalte zu verstehen, zueinander in Bezug und dann auch in irgendeiner Form umzusetzen. Es geht also nicht nur um schnell verwertbares Wissen, es sollte um Bildung im möglichst breiten Sinne gehen.

\section{Die Wege trennen sich früh}

Darum sollte es schließlich auch bereits in der schulischen Laufbahn gehen. Die Entwicklung der vergangenen Jahrzehnte geht allerdings in die andere Richtung und Entscheidungen, welchen Weg das Kind und der Jugendliche nehmen soll, müssen zu einer Zeit getroffen werden, wo meist noch gar nicht klar ist, wohin der Weg tatsächlich gehen soll oder kann. Nach vier Jahren gemeinsamer Volksschule erfolgt die erste Trennung und die Alternative zum Gymnasium ist im Allgemeinen mit einem Prestigeverlust verbunden. Nun also soll der Zugang zum Gymnasium wieder mit einer Aufnahmsprüfung geregelt werden. Mancher erinnert sich noch aus eigener Erfahrung in den 60er Jahren des 20. Jahrhunderts daran: Es war Stress pur. In Wahrheit war dieses Ereignis ein Stresstest und keine Überprüfung der Kenntnisse.

Aber die Gesamtschule zumindest bis zum Ende der achten oder neunten Schulstufe hat unter den derzeitigen politischen Vorzeichen wenig Aussicht auf baldige Verwirklichung. Dazu müsste man auch mehr tun, als nur ein neues Schildchen umhängen und schließlich auch die Lehrer anders ausbilden und bilden. Für Lehramtsstudien gilt ja seit dem Wintersemester 2014/15 eine Eignungsprüfung als Voraussetzung und zwar ohne Studienplatzbegrenzung. Ähnliches gibt es natürlich für die Kunstuniversitäten, um eine künstlerische Anlage als Voraussetzung festzustellen, auch wenn die Auswahl hier logischerweise alles andere als objektiv ist. Die tatsächliche Eignung und der Erfolg im Berufsleben erweist sich ohnedies erst in der Praxis und das zeigt sich nicht zuletzt daran, wieviel Freude in der Auseinandersetzung mit dem Metier, mit den Schülern und - siehe Medizin - mit den Patienten und Kollegen der Absolvent schließlich hat

meint Ihre

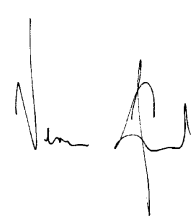

Verena Kienast

\section{Korrespondenzadresse}

\section{Kienast}

SpringerMedizin

Wien, Österreich

Verena.kienast@springer.at 